\title{
Electric-field-induced transport of microspheres in the isotropic and chiral nematic phase of liquid crystals
}

DOI:

10.1103/PhysRevE.95.022703

Document Version

Final published version

Link to publication record in Manchester Research Explorer

\section{Citation for published version (APA):}

Oh, J., Gleeson, H. F., \& Dierking, I. (2017). Electric-field-induced transport of microspheres in the isotropic and chiral nematic phase of liquid crystals. Physical review. E, Statistical physics, plasmas, fluids, and related interdisciplinary topics, 95, [022703]. https://doi.org/10.1103/PhysRevE.95.022703

\section{Published in:}

Physical review. E, Statistical physics, plasmas, fluids, and related interdisciplinary topics

\section{Citing this paper}

Please note that where the full-text provided on Manchester Research Explorer is the Author Accepted Manuscript or Proof version this may differ from the final Published version. If citing, it is advised that you check and use the publisher's definitive version.

\section{General rights}

Copyright and moral rights for the publications made accessible in the Research Explorer are retained by the authors and/or other copyright owners and it is a condition of accessing publications that users recognise and abide by the legal requirements associated with these rights.

\section{Takedown policy}

If you believe that this document breaches copyright please refer to the University of Manchester's Takedown Procedures [http://man.ac.uk/04Y6Bo] or contact uml.scholarlycommunications@manchester.ac.uk providing relevant details, so we can investigate your claim.

\section{OPEN ACCESS}




\title{
Electric-field-induced transport of microspheres in the isotropic and chiral nematic phase of liquid crystals
}

\author{
Jiyoung Oh, Helen F. Gleeson, ${ }^{*}$ and Ingo Dierking ${ }^{\dagger}$ \\ School of Physics and Astronomy, University of Manchester, Oxford Road, Manchester M13 9PL, United Kingdom
}

(Received 23 December 2016; published 23 February 2017)

\begin{abstract}
The application of an electric field to microspheres suspended in a liquid crystal causes particle translation in a plane perpendicular to the applied field direction. Depending on applied electric field amplitude and frequency, a wealth of different motion modes may be observed above a threshold, which can lead to linear, circular, or random particle trajectories. We present the stability diagram for these different translational modes of particles suspended in the isotropic and the chiral nematic phase of a liquid crystal and investigate the angular velocity, circular diameter, and linear velocity as a function of electric field amplitude and frequency. In the isotropic phase a narrow field amplitude-frequency regime is observed to exhibit circular particle motion whose angular velocity increases with applied electric field amplitude but is independent of applied frequency. The diameter of the circular trajectory decreases with field amplitude as well as frequency. In the cholesteric phase linear as well as circular particle motion is observed. The former exhibits an increasing velocity with field amplitude, while decreasing with frequency. For the latter, the angular velocity exhibits an increase with field amplitude and frequency. The rotational sense of the particles on a circular trajectory in the chiral nematic phase is independent of the helicity of the liquid crystalline structure, as is demonstrated by employing a cholesteric twist inversion compound.
\end{abstract}

DOI: 10.1103/PhysRevE.95.022703

\section{INTRODUCTION}

The transport of particles in liquids, initiated by applied electric fields, is generally known as electrophoresis and has been a longstanding topic of research in science. The phenomenon refers to the translational and rotational motion of particles under applied electric fields, and its importance in science is exemplified by a wide variety of applications in analytical chemistry, biology [1], and more recently also in modern display technologies [2]. The topic of particle suspensions in liquid crystals has seen an increasing amount of interest in recent years [3-5], which is due to the possibilities of tuning material properties [6], enhancing electro-optic devices [7], and generally producing materials with added functionality. Numerous different particles have been employed-dielectric [8,9], ferroelectric [10,11], and magnetic [12,13] nanoparticles, gold nanoparticles, which are incorporated into the mesogenic structures [14], onedimensional nanotubes in calamitic [15,16], discotic [17], ferroelectric [18], and lyotropic [19] phases, two-dimensional graphene-based materials, most often graphene oxide [20-22], just to name a few. Further materials are quantum dots [23], nanorods [24], and even biological structures [25]. Large nanoparticles and microparticles have been used to stabilize frustrated phases [26].

While phase behavior and material properties have played a major role in the investigated liquid crystalline systems, the underlying mechanism of particle translation and rotation by applied electric fields has been much less the focus of experimental investigations [[3] and references therein].

*Present address: School of Physics and Astronomy, University of Leeds, Leeds LS2 9JT, United Kingdom.

†Corresponding author: ingo.dierking@manchester.ac.uk
Nevertheless, this is an aspect of fundamental importance, because every application of particle-suspended liquid crystals will in one way or another depend on applied electric fields.

For nonmesogenic, isotropic liquids, respective studies go back for more than a century, when Weiler [27] reported a field-induced translation of chinin sulfate particles in turpentine oil, where the particle's velocity vector $\mathbf{v}$ is parallel to the direction of the applied electric field $\mathbf{E}$, with $\mathbf{v}=\mu \mathbf{E}$, where $\mu$ is the electrophoretic mobility. A few years later Quincke [28] observed the rotational motion of solid particles in a liquid subjected to a dc field. Only a century later theoretical models were developed which described translational motion [29-35] and Quincke rotation [36,37]. All of these are restricted to isotropic liquids, but they discuss different electric field forms and particle geometries, such as static [29] and alternating fields [30], spheroidal [31], and slender [32,33] particles, as well as particles with nonuniform charge distribution $[34,35]$. It is also worthwhile to distinguish between rotational motion of individual particles about their own axis, and particles moving on a circular trajectory within the fluid medium. In the present investigation we cannot comment on the former type of motion, as this cannot be visualized with the particles employed. We here concentrate on macroscopic trajectories of microparticles within the liquid crystal.

Investigations of microparticles in liquid crystals have often been focused on the regime of relatively large volume fractions, for example, in the decoration of defects and trapping at interfaces [38]. Such particle concentrations do not take into account the fluid dynamical aspects of the liquid crystal, where the flow field of one particle can disturb the trajectory of another over distances of several tens of micrometers. Similarly, investigations on smectic phases have mostly been nonsystematic $[39,40]$, with more comprehensive 
experimental accounts for nematic and smectic phases given in $[8,9,41]$, while still being descriptive in the discussion of trends, as up to date there does not seem to be a theoretical description available for the electric-field-induced motion of particles in liquid crystalline phases. The present study concentrates on possible effects of chirality and reports results of systematic investigations related to the isotropic and the chiral nematic phase of liquid crystals.

\section{EXPERIMENTAL}

The liquid crystal employed in this study was first synthesized by Walba et al. and abbreviated as W46, also commercially available from Aldrich as $(S, S)$-EPHDBPE (short for 4[(S,S)-2,3-epoxyhexyloxy]-phenyl-4-(decyloxy)-benzoate). It has a phase sequence on heating of Cryst. $77 \mathrm{~N}^{*} 95.7 \mathrm{I}$, while on cooling monotropic smectic phases are observed: I $95.7 \mathrm{~N}^{*}$ (78.6 SmC*) (57.2 SmI*) 57 Cryst., with all temperatures in degrees centigrade. The interesting property of this compound is its temperature-induced change of the helical twist sense of the cholesteric phase at about $82.5^{\circ} \mathrm{C}$ [42] from left- to right-handed at increasing temperature. To our knowledge the dielectric properties of this material are not investigated in detail. We found that the dielectric anisotropy in the cholesteric phase is approximately $\Delta \varepsilon=2$ at $10 \mathrm{kHz}$.

Commercial spacer beads, untreated, monodisperse spherical $\mathrm{SiO}_{2}$ microparticles of diameter $2.5 \mu \mathrm{m}$, were placed at the entrance of sandwich cells (E.H.C., Japan, planar boundary conditions, antiparallel rubbing) with a gap between 4 and $10 \mu \mathrm{m}$ to be dragged into the electrode area when capillary filling the cell with the liquid crystal. The cells had very welldefined gaps $( \pm 0.2 \mu \mathrm{m})$, and different cell gaps were employed for different experiments. The preparation procedure assured most of the time the presence of single microparticles, far away from other particles, to reduce mutual influences of the flow fields during motion. It was furthermore found, independent of a cell gap, that the dispersed microspheres were located approximately in the middle of the cell gap, not touching either substrate, as far as could be confirmed by changing the focus of a $x 20$ objective. We could not observe any motion of the particles towards either of the substrates under field application. Nevertheless, it should also be pointed out that this would be hard to quantify with the objectives available. Electric square-wave fields were applied in the frequency range between 2 and $400 \mathrm{~Hz}$ at amplitudes between 0 and $30 V_{p p}$, by use of a TTi TG1010 function generator in combination with an in-house-built power amplifier.

Samples were investigated in a polarizing microscope (Olympus BH-2) equipped with a Linkam hot stage and temperature controller for control of relative temperatures to within $\pm 0.1 \mathrm{~K}$. A video camera (uEye UI-5460-C), connected to a computer, allowed the recording of movies of particle motion at a maximum frame rate of $57 \mathrm{fps}$. Individual frames were extracted from the movies for analysis with ImageTool 3.0, developed at the University of Texas Health Science Center, San Antonio, TX. The conversion factor from pixels to micrometers was 0.278 , so that errors in distance measurements can be estimated to approximately $\pm 0.5 \mu \mathrm{m}$.

\section{RESULTS AND DISCUSSION}

\section{A. Isotropic phase}

For particles dispersed in the isotropic phase of a liquid crystal, one can observe several different modes of motion, depending on both applied electric field amplitude $E$ and frequency $f$. For this case it is clearly irrelevant if the investigated molecules are chiral or nonchiral. Also, no differences in behavior were observed over a temperature regime up to $25 \mathrm{~K}$ into the isotropic phase, so that for the following experiments a fixed temperature of $110^{\circ} \mathrm{C}, 13 \mathrm{~K}$ above the clearing temperature, was chosen. It is anticipated that this temperature is far enough into the isotropic phase region so that residual liquid crystalline order at the boundaries, which may influence the experiment, has vanished. The different regimes of translational particle behavior are depicted in Fig. 1(a). Below a certain threshold electric field, in this case

(a)
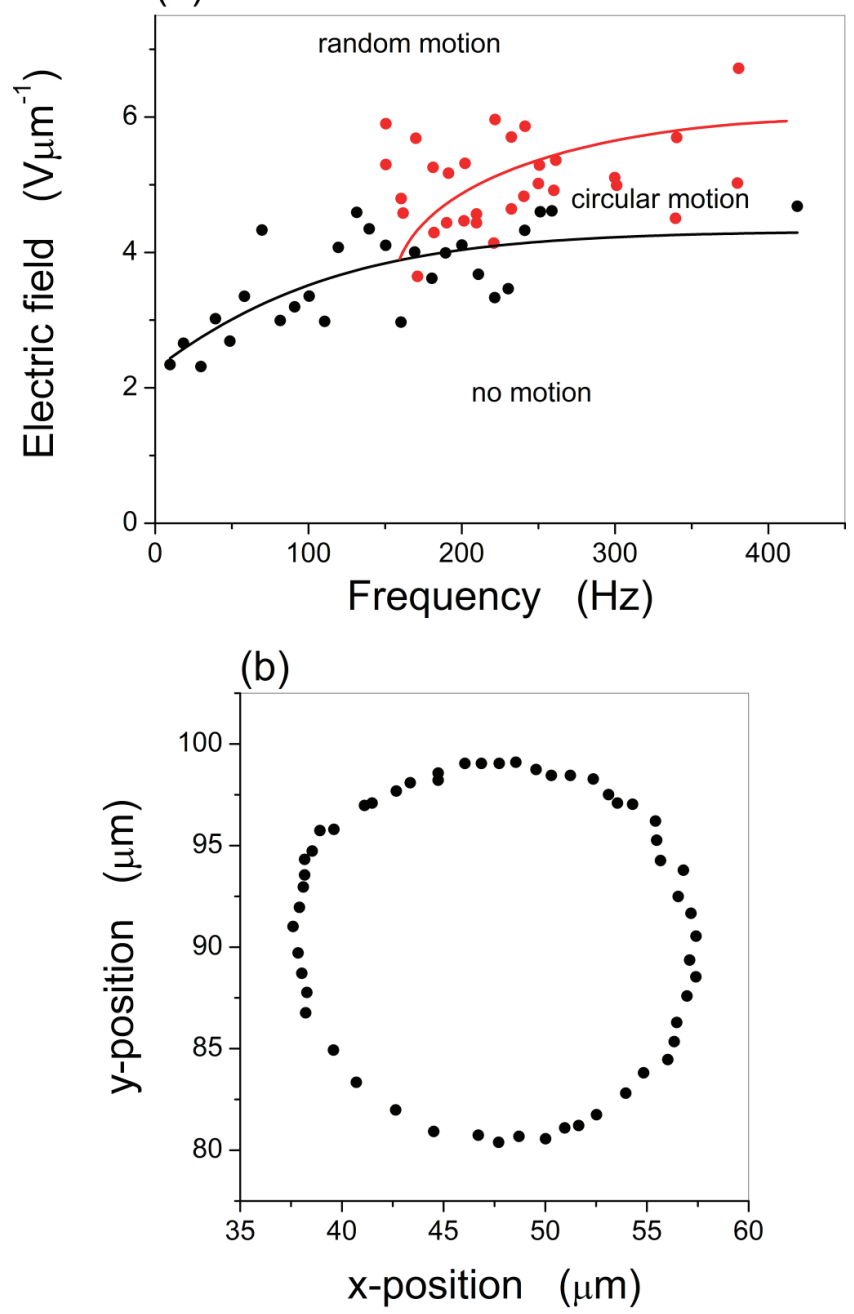

FIG. 1. (a) Field amplitude-frequency stability regimes for circular and random motion of microspheres in the isotropic phase of a liquid crystal. (Black and red symbols indicate the boundaries between different motional behavior, lines are a guide to the eye.) (b) Exemplary circular microsphere trajectory in the plane of the liquid crystal sandwich cell. 
(a)

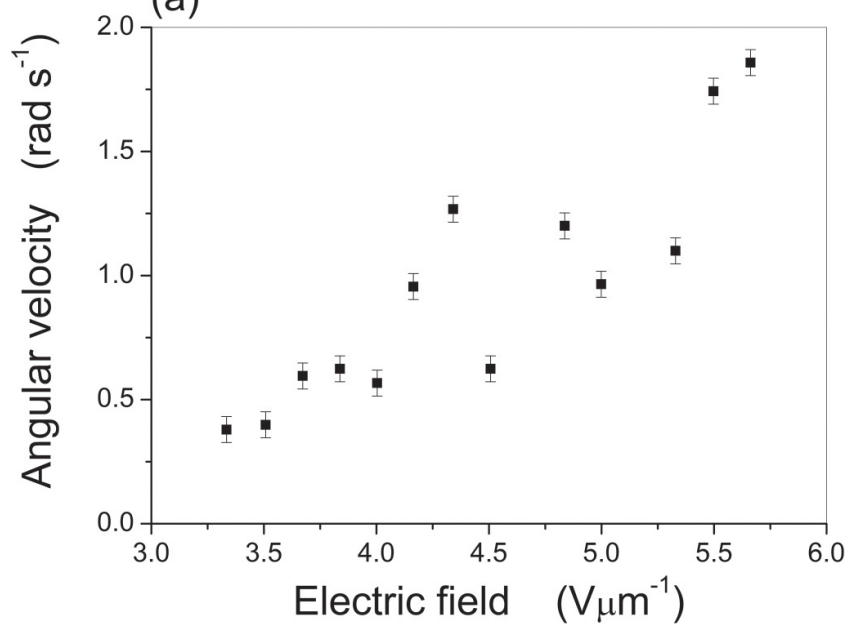

(b)

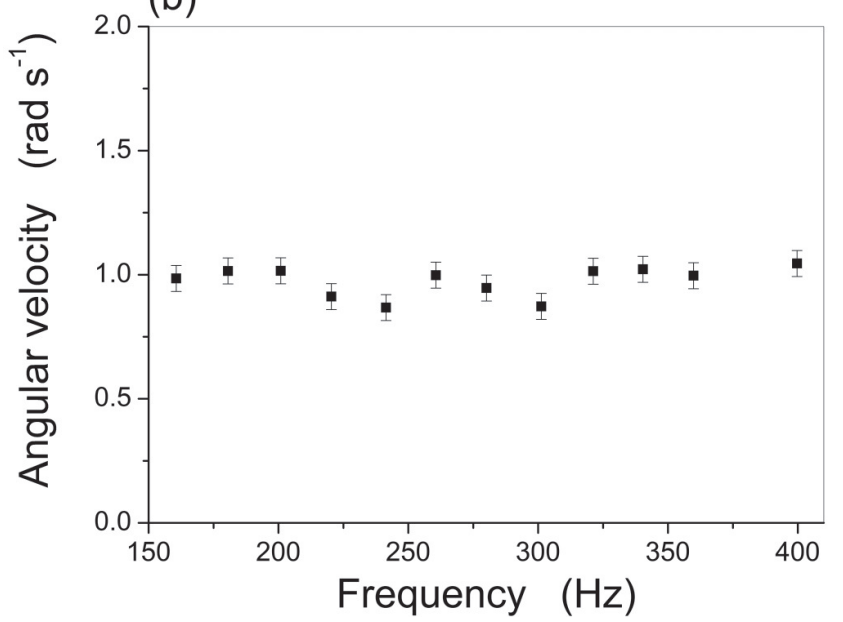

FIG. 2. Angular microsphere velocity as a function of (a) applied electric field amplitude at a constant frequency of $f=160 \mathrm{~Hz}$, and (b) frequency at constant electric field amplitude of $E=5 \mathrm{~V} / \mu \mathrm{m}$. The angular velocity $\omega$ increases with increasing field and is independent of frequency.

approximately $2 \mathrm{~V} \mu \mathrm{m}^{-1}$, no particle motion is observed at all. This threshold field slightly increases as the frequency is increased. Above the threshold the particles move on a circular trajectory in the plane of the sandwich cell, with a velocity vector perpendicular to the electric field. This behavior can thus not be described by classical electrophoresis, where the motion is in the direction of the applied electric field. An exemplary particle trajectory is shown in Fig. 1(b), which exhibits a diameter of about $20 \mu \mathrm{m}$, much larger than the size of the particle itself. The circular particle motion is observed in a relatively narrow band of field amplitude and frequency, which widens for increasing $E$ and $f$. Further increase of field and frequency then results in random particle motion, which can be attributed to the onset of electrohydrodynamic convection and material flow.

Figure 2 depicts the results of an investigation of the dependence of the angular velocity $\omega$ on (a) electric field amplitude at constant frequency $f=160 \mathrm{~Hz}$ and (b) frequency at constant electric field of $E=5 \mathrm{~V} / \mu \mathrm{m}$, within the regime (a)

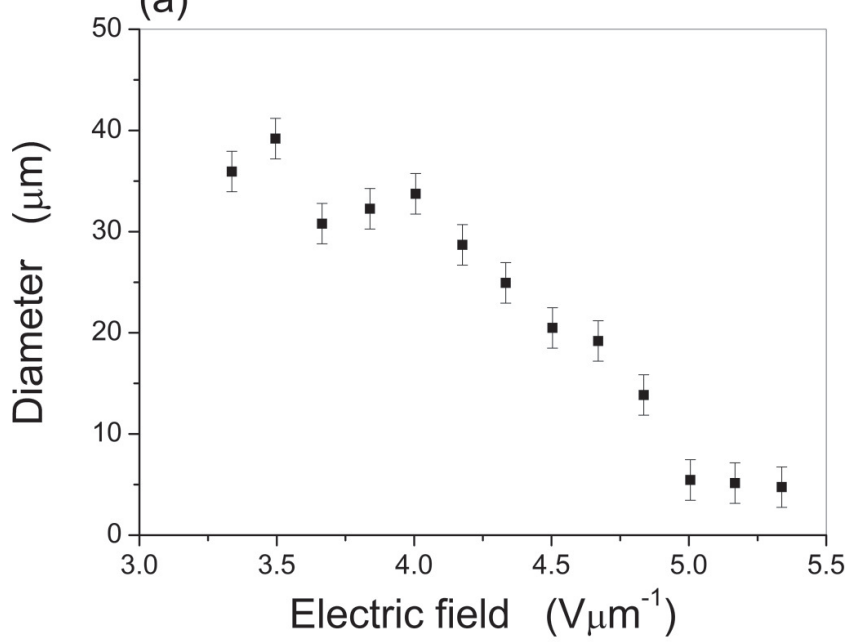

(b)

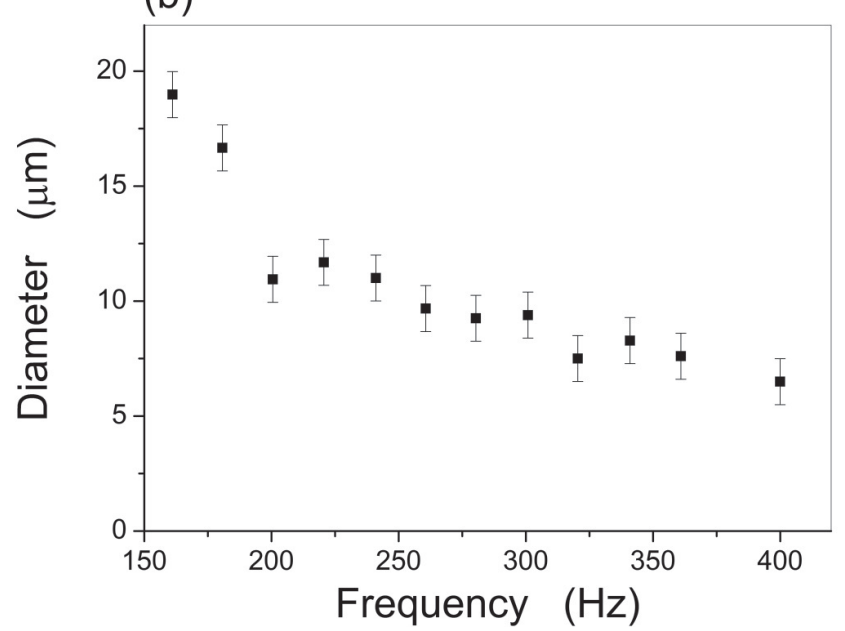

FIG. 3. Diameter of the circular microsphere trajectory as a function of (a) electric field amplitude and (b) frequency, for the same experimental conditions as in Fig. 2. For both parameters the diameter decreases for increasing control parameter.

of circular particle trajectories. The angular velocity of the particles increases practically linearly with applied field $E$ and is independent of frequency, i.e., $\omega(E, f)=\omega(E)$. At the same time, the diameter $d$ of the circular trajectory decreases in first approximation linearly with increasing electric field amplitude [Fig. 3(a), $f=160 \mathrm{~Hz}$ ] and decreases slightly with respect to a change in frequency [Fig. 3(b)], $E=5 \mathrm{~V} / \mu \mathrm{m}$ ). The strongest decrease is observed for low frequencies where ionic motion still influences the particle behavior significantly $(f<200 \mathrm{~Hz})$, while the diameter approaches a constant value comparable to the cell gap for frequencies above the regime of influence of ionic motion $(f>400 \mathrm{~Hz})$.

The particle motion observed in the isotropic phase without any effects of electrohydrodynamic turbulence is circular in nature, in the plane of the substrates and perpendicular to the applied electric field. It occurs in a regime of frequency and field amplitude above a certain critical threshold field $E_{\text {cr }}$ and possesses some similarity with Quincke rotation [28]. Following a study of Liao et al. [8], although carried out in a 


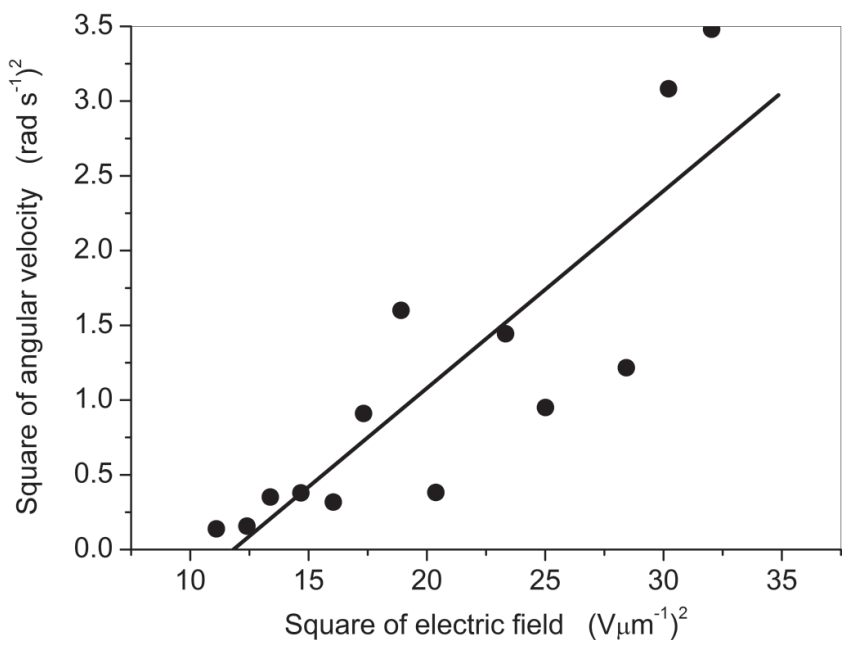

FIG. 4. A fit of the experimental data for the isotropic phase to a rearranged Eq. (1) yields a critical field of approximately $E_{\mathrm{cr}} \approx$ $3 \mathrm{~V} / \mu \mathrm{m}$ and a Maxwell-Wagner relaxation time of about $\tau_{\mathrm{MW}} \approx 1 \mathrm{~s}$, in agreement with qualitative experimental observations.

smectic, not an isotropic system, they associated the critical field with the angular velocity $\omega$ via

$$
\omega=\frac{1}{\tau_{\mathrm{MW}}} \sqrt{\frac{E^{2}}{E_{\mathrm{cr}}^{2}}-1,}
$$

with $\tau_{\mathrm{MW}}$ the Maxwell-Wagner interfacial polarization relaxation time and $E$ the applied electric field amplitude. A fit of the data of Fig. 2(a) to a rearranged Eq. (1), depicted

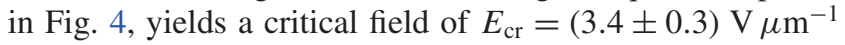
and $\tau_{\mathrm{MW}}=(0.81 \pm 0.27) \mathrm{s}$, which is in very good agreement with the observation that no particle motion is detected below approximately $3 \mathrm{~V} \mathrm{\mu m}^{-1}$. The Maxwell-Wagner relaxation time can also be determined from the dielectric and conductive properties of the liquid crystal (LC) and the microsphere $(\mathrm{SiO})$ :

$$
\tau_{\mathrm{MW}}=\frac{\left(\varepsilon_{\mathrm{SiO}}+2 \varepsilon_{\mathrm{LC}}\right)}{\left(\sigma_{\mathrm{SiO}}+2 \sigma_{\mathrm{LC}}\right)}
$$

with $\varepsilon_{\mathrm{SiO}}$ and $\varepsilon_{\mathrm{LC}}$ the permittivities of the microsphere and the liquid crystal, and $\sigma_{\mathrm{SiO}}$ and $\sigma_{\mathrm{LC}}$ the respective conductivities. For typical values of $\varepsilon_{\mathrm{SiO}} \approx 3.9, \varepsilon_{\mathrm{LC}} \approx 5, \sigma_{\mathrm{SiO}}<10^{-12} \mathrm{Sm}^{-1}$, and $\sigma_{\mathrm{LC}} \approx 10^{-10}$, one estimates a relaxation time in the order of $\tau_{\mathrm{MW}} \approx 1 \mathrm{~s}$, as observed in the experiment.

The critical field can further be used to estimate the viscosity of the liquid crystal in its isotropic phase, according to

$$
E_{\mathrm{cr}}^{2}=\gamma \frac{\sigma_{\mathrm{LC}}}{\epsilon_{0}^{2} \epsilon_{\mathrm{LC}} \varepsilon_{\mathrm{SiO}}}
$$

for $\sigma_{\mathrm{LC}} \gg \sigma_{\mathrm{SiO}}$ and $\varepsilon_{\mathrm{LC}} \approx \varepsilon_{\mathrm{SiO}}$, and where $\gamma$ is the viscosity, and $\varepsilon_{0}$ is the permittivity of vacuum, and $8.8 \times 10^{-12} \mathrm{~F} \mathrm{~m}^{-1}$. Again, using typical values, one obtains for the liquid crystal viscosity $\gamma \approx 0.2 \mathrm{Pas}$ in the isotropic phase. This value is in reasonable agreement with expected values for the isotropic phase of a liquid crystal, given the experimental uncertainties. It should be noted that viscosity measurements are notoriously difficult, with a large range of values quoted in literature. Also, the uncertainty about the conductivity value could be quite substantial, which could easily vary by a factor of
10. The model of Quincke rotation appears to explain the observed circular particle motion quite well, certainly on a qualitative basis. However, one should also note that the system is rather constrained, so that influences of the substrates cannot completely be ruled out. This can, for instance, be inferred from laser tweezing experiments, where particles need to be trapped at least tens of micrometers away from substrates to avoid surface effects $[43,44]$.

At last, one may discuss the frequency dependence of circular microparticle motion. At a fixed electric field amplitude, the diameter of the circular particle trajectory decreases for increasing field frequency (Fig. 3). Beyond approximately $400 \mathrm{~Hz}$, the trajectory diameter tends toward values of twice the particle diameter, such that an actual particle motion can be considered to cease. Such a frequency $f_{\max }$ has also been observed in other systems [45-47] and can be related to a delay time $\tau_{\text {del }}$ :

$$
f_{\max }=\frac{1}{\tau_{\mathrm{del}}},
$$

where the field frequency is faster than the delay time for the microsphere to respond. In the present case the delay time is about $\tau_{\mathrm{del}} \approx 2.5 \times 10^{-3} \mathrm{~s}$. For the description of the present system, also ionic diffusion is of importance. This is characterized by an ion diffusion coefficient $D$ and the sphere radius $r$, by Schwarz' formula

$$
\tau_{\mathrm{del}}=\frac{r^{2}}{2 D}
$$

With the values above, an ion diffusion coefficient of $\mathrm{D} \approx$ $3 \times 10^{-10} \mathrm{~m}^{2} \mathrm{~s}^{-1}$ is obtained for the isotropic phase, that is slightly smaller than those in water $\left(\sim 5-10 \times 10^{-10} \mathrm{~m}^{2} \mathrm{~s}^{-1}\right)$, which can be understood when considering the size of a liquid crystal molecule.

\section{B. Chiral nematic phase}

The stability diagram for the chiral nematic or cholesteric phase is slightly more complicated than the one for the isotropic liquid and is depicted in Fig. 5. Again, there is no particle motion to be observed below a frequency-dependent threshold electric field. Nevertheless, before the regime of circular trajectories is reached, one observes a certain range of field amplitudes and frequencies, where the particle motion follows a linear trajectory, as it was reported for the achiral nematic phase [9]. At large electric field strengths again a random motion of particles is observed which can be attributed to electrohydrodynamic flow. The linear motion of a microscopic sphere is demonstrated in Fig. 6 for a particle well removed of any other particles, dust, or defects at $f=40 \mathrm{~Hz}$ and $E=2.7 \mathrm{~V} / \mu \mathrm{m}$. An investigation of the linear particle motion as a function of electric field amplitude [Fig. 7(a) at constant frequency $f=40 \mathrm{~Hz}$ ] and frequency [Fig. 7(b) at constant electric field $E=2.7 \mathrm{~V} / \mu \mathrm{m}$ ] suggests an approximately linear increase of the particle velocity with

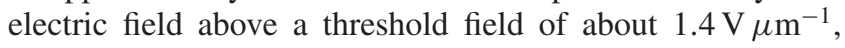
while the frequency dependence is rather insignificant. This process is reminiscent of electrophoresis, and we can calculate an electrophoretic mobility $\mu_{e} \approx 5 \mu \mathrm{m}^{2} \mathrm{~V}^{-1} \mathrm{~s}^{-1}$ from the linear relation between velocity and field, $v=\mu_{e} E$, above 


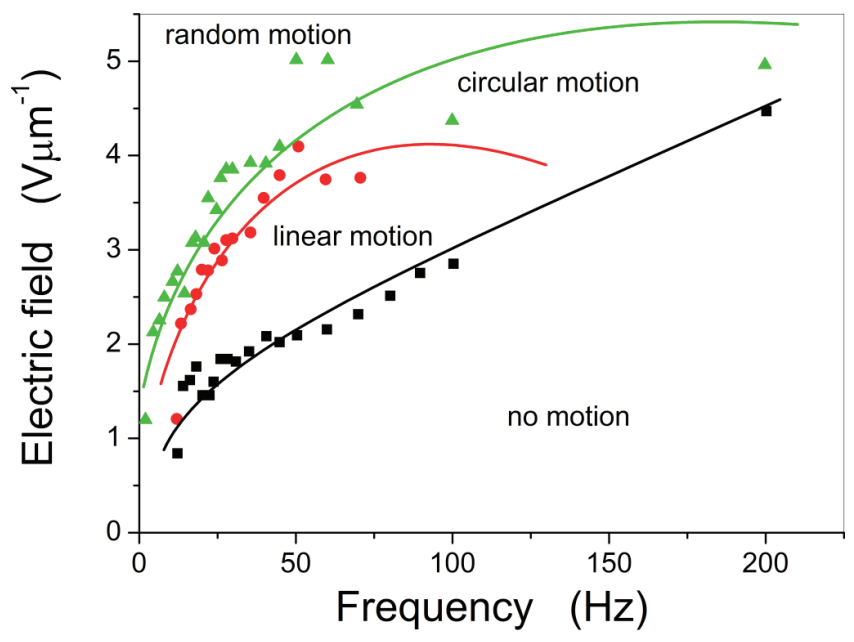

FIG. 5. Field amplitude-frequency stability regimes for linear, circular, and random motion of microspheres in a chiral nematic liquid crystal phase. (Black, red, and green symbols indicate the boundaries between different motional behavior, lines are a guide to the eye.)

the threshold. Fitting the dependence of linear velocity as a function of electric field by a nonlinear relationship $v=$ $a\left(E-E_{\mathrm{cr}}\right)+b\left(E-E_{\mathrm{cr}}\right)^{3}$, as proposed by Ryzhkova et al. [48], does not result in any improved description of the data. It can therefore be concluded that the regime termed a linear particle motion regime is that of classical electrophoresis, albeit motion does not occur in the direction of the applied electric field. This scenario, which is not possible in an isotropic liquid, is indeed possible in a liquid crystal, as pointed out by Lavrentovich [3]. Here, the velocity vector does not need to be parallel to an applied electric field but may have an arbitrary direction, thus also perpendicular to the electric field direction.

The regime of circular particle motion is illustrated by the behavior depicted in Fig. 8 at $f=40 \mathrm{~Hz}$ and $E=$

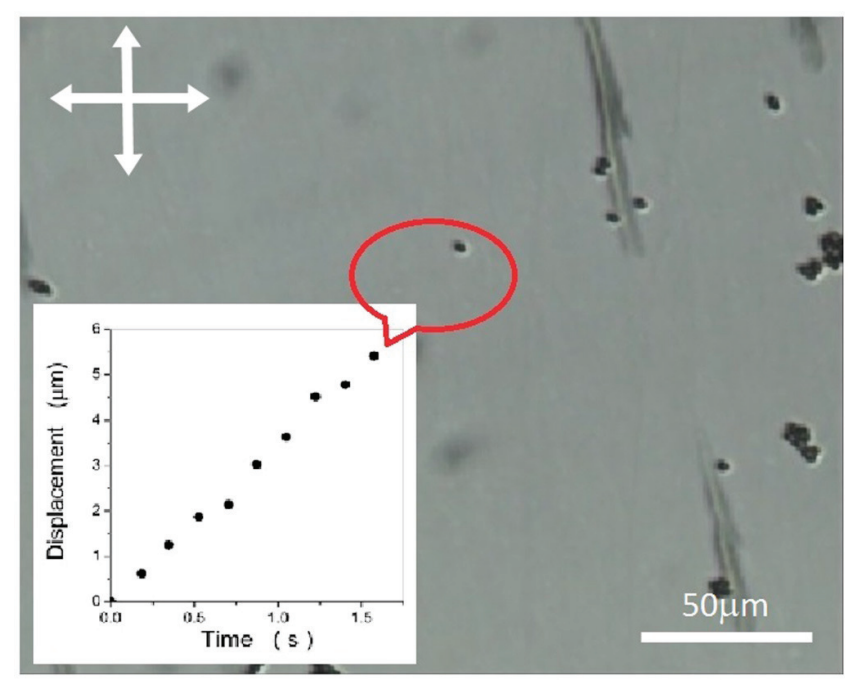

FIG. 6. Exemplary trajectory of a microsphere in the chiral nematic phase, subjected to relatively small fields at low frequencies ( $E=2.7 \mathrm{~V} / \mu \mathrm{m}, f=40 \mathrm{~Hz}$ ), leading to linear particle motion.

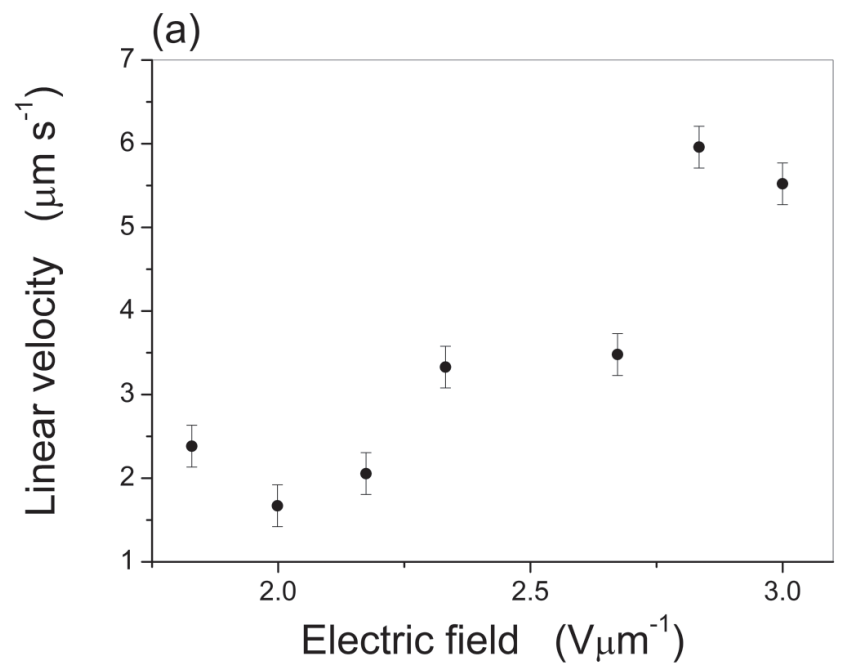

(b)

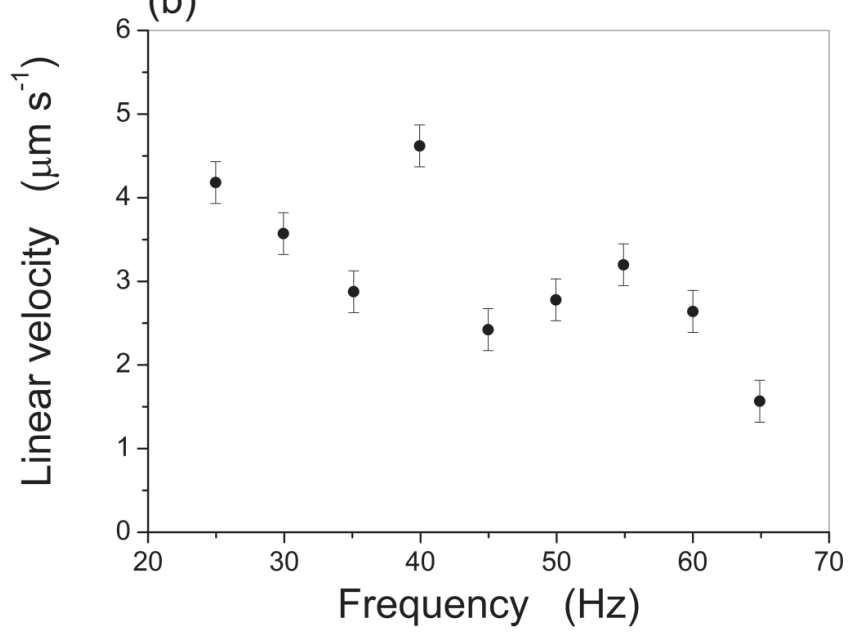

FIG. 7. Linear microsphere velocity as a function of (a) electric field amplitude and (b) frequency, in the chiral nematic phase. While the velocity increases with increasing field, it decreases slightly with increasing frequency, which is in qualitative agreement with the particle motion observed in achiral nematic phases [9].

$3.9 \mathrm{~V} / \mu \mathrm{m}$. In the texture the onset of a structure due to electrohydrodynamic effects is observed. It should be pointed out that all measurements were performed at electric fields smaller than those leading to electrohydrodynamic domains. At the applied fields the Grandjean orientation was always assured. In Fig. 8, the particle is in the vicinity of other microspheres, as well as a dust particle and remains of an oily streaks defect. This is most likely the reason for the slight deviation of the particle trajectory from a completely circular shape. Also here, the angular velocity increases with increasing electric field amplitude, as was the case for the isotropic phase, even at a comparable threshold field of about $3.2 \mathrm{~V} \mathrm{\mu m}^{-1}$ [Fig. 9(a), $f=40 \mathrm{~Hz}$ ]. The dependence of the angular velocity on the frequency of the applied field is still not markedly pronounced [Fig. 9(b), $E=3.6 \mathrm{~V} / \mu \mathrm{m}$ ].

Following a similar discussion as above for the isotropic phase, we can fit the data of Fig. 9(a) for the cholesteric phase 


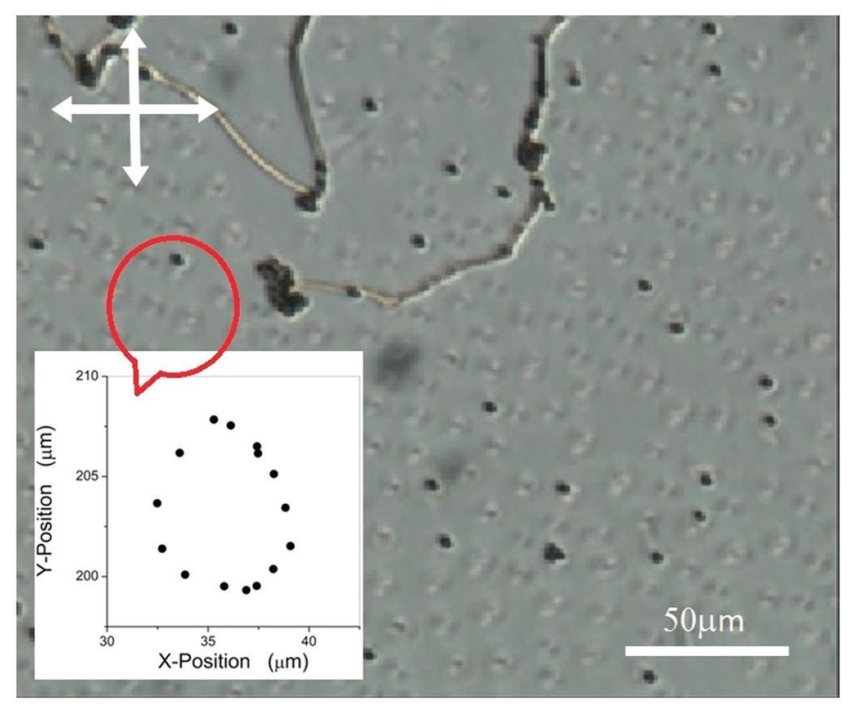

FIG. 8. Exemplary trajectory of a microsphere in the chiral nematic phase, observed in the plane of the sandwich cell at slightly increased electric field amplitude as compared to Fig. 6 $(E=3.9 \mathrm{~V} / \mu \mathrm{m}, f=40 \mathrm{~Hz}$ ), leading to circular particle motion. The onset of a domain structure in the texture indicates the onset of electrohydrodynamic effects in the liquid crystal. All electric-fielddependent measurements were performed below this onset.

to a rearranged version of Eq. (1):

$$
\omega^{2}=\left(\frac{1}{\tau_{\mathrm{MW}}^{2}}\right)\left(\frac{E^{2}}{E_{\mathrm{cr}}^{2}}-1\right) .
$$

This is depicted in Fig. 10, and we obtain the critical field to $E_{\mathrm{cr}}=3.1 \pm 0.1 \mathrm{~V} \mathrm{\mu m}^{-1}$ and the Maxwell-Wagner relaxation time to $\tau_{\mathrm{MW}}=3.9 \pm 0.14 \mathrm{~s}$. Qualitatively these results agree well with our observations that there is no particle motion

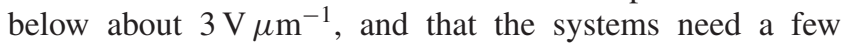
seconds to respond when first applying an electric field.

Estimating the ion diffusion coefficient via Eqs. (4) and (5), one obtains a value of $D \approx 8 \times 10^{-11} \mathrm{~m}^{2} \mathrm{~s}^{-1}$. This is much smaller than the value observed for the isotropic phase, which is accounted for by an increase in viscosity, and especially also due to the fact that we are now investigating a self-organized and ordered liquid crystal system, which additionally also exhibits a helical superstructure.

\section{Dependence on chirality}

One of the interesting questions is: if and possibly how does the circular motion of microspheres depend on the chirality of the liquid crystal system, i.e., the handedness of the cholesteric phase. For that matter, we have employed a so-called twist inversion compound [42], a cholesteric single-component system which changes handedness solely by changing temperature. This is demonstrated by a series of textures shown in Fig. 11, starting in (a) the crystalline phase and (b) increasing temperature into the left-handed cholesteric texture with pitch changes close to the twist inversion temperature, (c) the re-formation of the cholesteric helix after the inversion into a right-handed structure, accompanied by oily streaks (a)

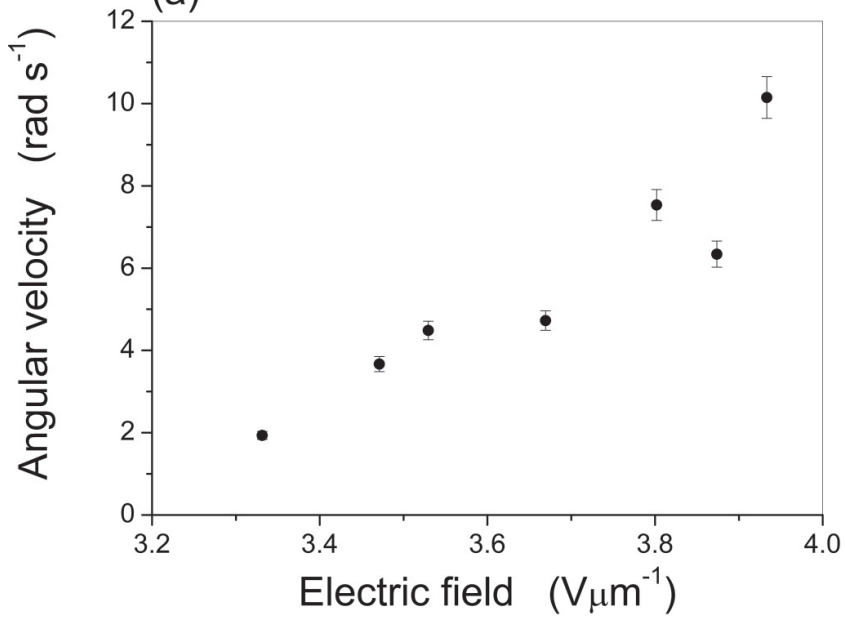

(b)

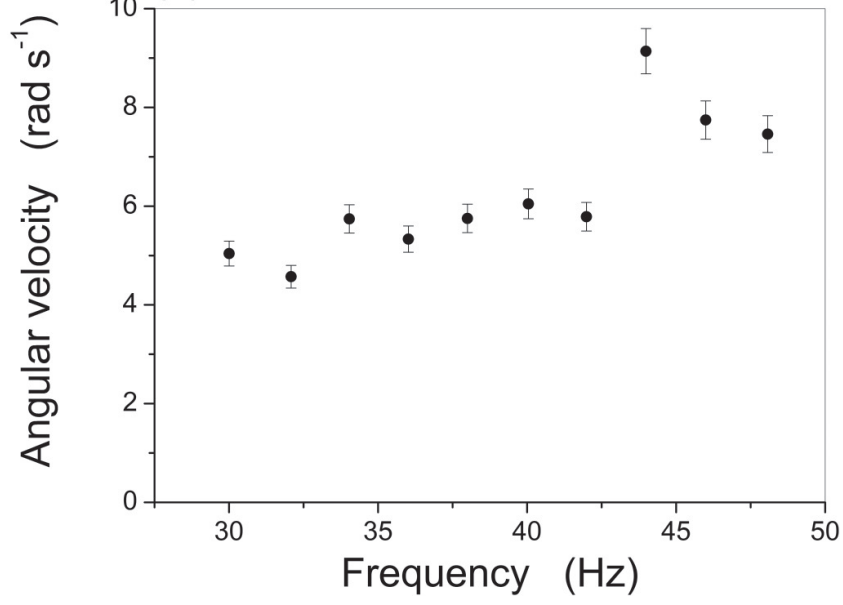

FIG. 9. Angular microsphere velocity in the chiral nematic phase as a function of (a) electric field amplitude at constant frequency of $f=40 \mathrm{~Hz}$ and (b) frequency at applied field $E=3.6 \mathrm{~V} / \mu \mathrm{m}$. For increasing control parameters the angular velocity increases in magnitude.

defects, which (d) anneal into the planar Grandjean texture at even higher temperatures. The corresponding temperature dependence of the pitch $P(T)$, determined by two independent methods, is shown in part (e) of Fig. 11.

Carrying out the experiment five times in both the lefthanded $\left(T=81^{\circ} \mathrm{C}, P=-7.2 \mu \mathrm{m}\right)$ as well as the righthanded $\left(T=87^{\circ} \mathrm{C}, P=2.4 \mu \mathrm{m}\right)$ cholesteric structure provided no evidence that the clockwise or counterclockwise rotation of the microspheres along the circular trajectory is in any way related to the handedness of the liquid crystal helical superstructure. This is due to the fact that the spherical shape of the microparticles is inherently nonchiral in nature and thus cannot experience any chirality field that it is dispersed in. We would anticipate a different result for particles of a chiral shape, such as propellers, for instance. Similarly, one would not expect any significant influence of the absolute value of the pitch $P$ on the motion of microspheres, as a spherical particle would not be expected to couple with the surrounding chiral field of the cholesteric liquid crystal matrix it is embedded 


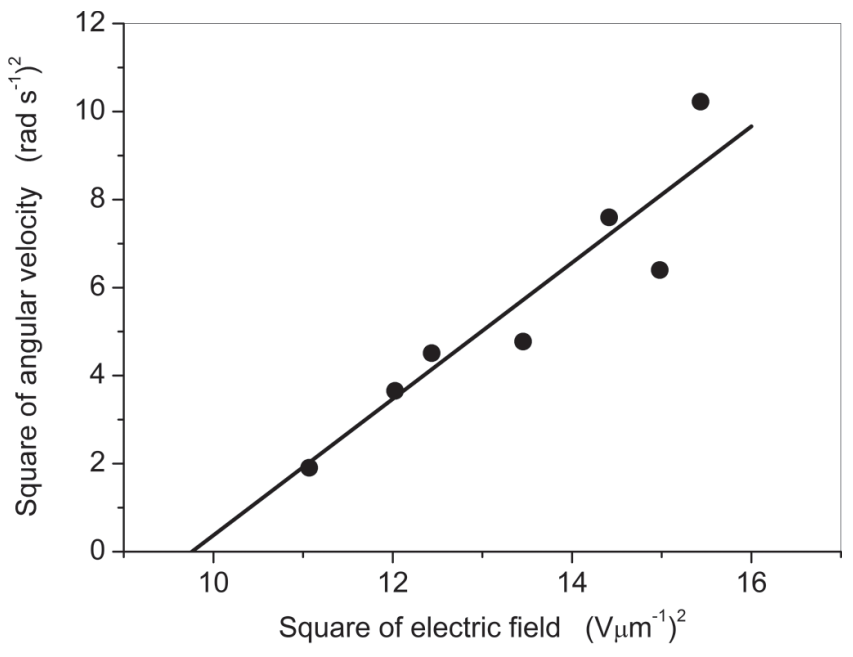

FIG. 10. A fit of the experimental data for the chiral nematic phase to a rearranged Eq. (1) yields a critical field, which is still comparable to that of the isotropic phase, $E_{\mathrm{cr}} \approx 3 \mathrm{~V} / \mu \mathrm{m}$, but with an increased Maxwell-Wagner relaxation time to $\tau_{\mathrm{MW}} \approx 4 \mathrm{~s}$.

in. Thus we did not investigate the particle motion as a function of helical pitch (parameterized by temperature) in any quantitative detail. Nevertheless, qualitatively no difference in motion was observed for a pitch (i) approximately 3 times larger $\left(T=81^{\circ} \mathrm{C}, P=7.2 \mu \mathrm{m}\right)$ than or (ii) comparable $(T=$ $\left.87^{\circ} \mathrm{C}, P=2.4 \mu \mathrm{m}\right)$ to the particle size. Again, this would expected to be different for chiral-shaped microparticles.

\section{CONCLUSIONS}

The translational motion of microspheres in the isotropic and chiral nematic phase of a liquid crystal was investigated through application of an electric field with varying amplitude and frequency. The stability regimes for circular, linear, and random motion of the particles were determined, and linear as well as angular velocities measured as a function of electric field amplitude and applied frequency. The motion of particles in the isotropic phase is well explained by Quincke rotation. From an analysis the critical electric field was determined to

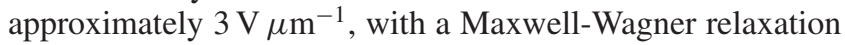
time of the order of a few seconds. The ion diffusion coefficient $D$ is of the order $D \approx 10^{-10} \mathrm{~m}^{2} \mathrm{~s}^{-1}$, and slightly smaller than that of water, which can be explained by the relative size of the liquid crystal molecules as compared to water molecules. In the chiral nematic phase the critical field is comparable to that of the isotropic phase, while the Maxwell-Wagner relaxation time is somewhat longer. The electrophoretic

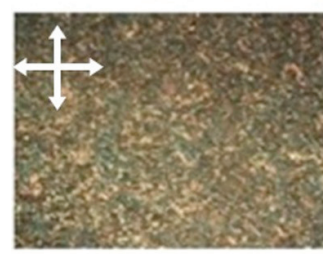

(a) $\mathrm{C}$ at $60^{\circ} \mathrm{C}$

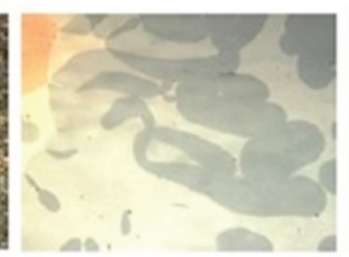

(b) $\mathrm{N}^{*}$ at $81.5^{\circ} \mathrm{C}$

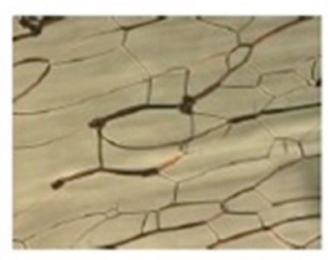

(c) $\mathrm{N}^{*}$ at $87.2^{\circ} \mathrm{C}$

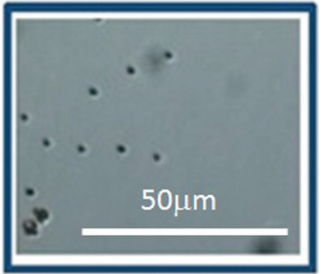

(d) $\mathrm{N}^{*}$ at $90.3^{\circ} \mathrm{C}$

(e)

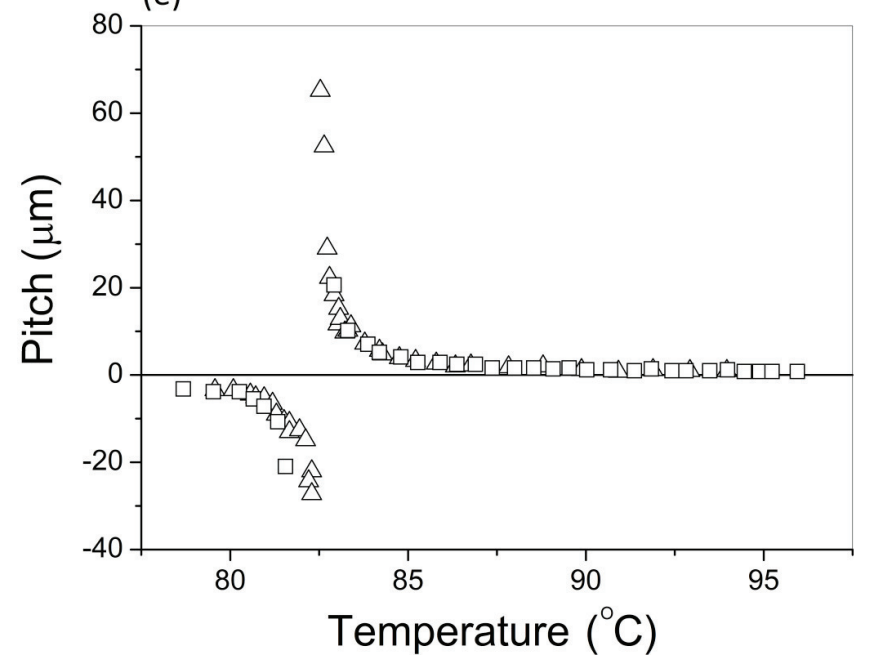

FIG. 11. Texture series of the employed cholesteric twist inversion compound W46 to establish a possible relationship between direction of circular particle motion and helix sense. (a) Crystalline phase, (b) left-handed cholesteric phase with pitch jumps approaching the twist inversion temperature, (c) right-handed cholesteric phase with remains of oily streaks formed during the twist inversion, and (d) annealed right-handed cholesteric phase in Grandjean orientation. The cell gap is $d=6 \mu \mathrm{m}$, planar anchoring conditions. Part (e) demonstrates the helical pitch inversion as a function of temperature, with negative values implying a left-handed helix and positive values a right-handed structure (after [42], reproduced by permission of Taylor \& Francis). 
motion of the particle in the regime of linear translation was found to depend linearly on the applied field with an electrophoretic mobility of the order of $\mu_{e} \approx 5 \mu \mathrm{m}^{2} \mathrm{~V}^{-1} \mathrm{~s}^{-1}$. The ion diffusion coefficient of the chiral nematic phase, $D \approx 8 \times 10^{-11} \mathrm{~m}^{2} \mathrm{~s}^{-1}$, is approximately 1 order of magnitude smaller than that of the isotropic phase. This can not only be accounted for by the temperature dependence of the viscosity, but implies further effects that increase the viscosity, such as self-organization, ordering, and possibly the formation of the helical superstructure of the cholesteric phase. A further contribution could lie in the fact that in the liquid crystal phase defects are formed in the vicinity of particles, thus effectively increasing its interaction size or radius of gyration. At last, the sense of circular particle trajectories is independent of the handedness of the chiral nematic helical superstructure, as investigated for a twist inversion compound. This is due to the fact that the spherical microparticles employed in this study are inherently nonchiral and cannot experience a surrounding chirality field. A different observation would be expected for chiral microparticles.
[1] D. M. Hawcroft, Electrophoresis: The Basics (Oxford University Press, Oxford, 1997).

[2] S.-T. Wu and D.-K. Yang, Reflective Liquid Crystal Displays (Wiley, Chichester, 2001), Chap. 1.

[3] O. D. Lavrentovich, Soft Matter 10, 1264 (2014).

[4] J. P. F. Lagerwall and G. Scalia, Current Appl. Phys. 12, 1387 (2012).

[5] Y. Bai and N. L. Abbott, Langmuir 27, 5719 (2011).

[6] O. Stamatoiu, J. Mirzaei, X. Feng, and T. Hegmann, in Liquid Crystals: Materials Design and Self-Assembly, edited by C. Tschierske, Topics in Current Chemistry Vol. 318 (Springer, New York, 2012), p. 331.

[7] H. Qi and T. Hegmann, J. Mater. Chem. 18, 3288 (2008).

[8] G. Liao, I. I. Smalyukh, J. R. Kelly, O. D. Lavrentovich, and A. Jakli, Phys. Rev. E 72, 031704 (2005).

[9] I. Dierking, G. Biddulph, and K. Matthews, Phys. Rev. E 73, 011702 (2006).

[10] Y. Reznikov, O. Buchnev, O. Tereshchenko, A. Glushchenko, and J. West, Appl. Phys. Lett. 82, 1917 (2003).

[11] A. Glushchenko, C. Il Cheon, J. West, F. H. Li, E. Buyuktanir, Y. Reznikov, and A. Buchnev, Mol. Cryst. Liq. Cryst. 453, 227 (2006).

[12] N. Podoliak, O. Buchnev, O. Buluy, G. D'Alessandro, M. Kaczmarek, Y. Reznikov, and T. J. Sluckin, Soft Matter 7, 4742 (2011).

[13] A. Mertelj, N. Osterman, D. Lisjak, and M. Copic, Soft Matter 10, 9065 (2014).

[14] T. Hegmann, H. Qi, and V. M. Marx, J. Inorg. Organomet. Polym. Mater. 17, 483 (2007).

[15] I. Dierking, G. Scalia, and P. Morales, J. Appl. Phys. 97, 044309 (2005).

[16] J. P. F. Lagerwall and G. Scalia, J. Mater. Chem. 18, 2890 (2008).

[17] S. Kumar and H. K. Bisoyi, Angew. Chem. Int. Ed. 46, 1501 (2007).

[18] M. Yakemseva, I. Dierking, N. Kapernaum, N. Usoltseva, and F. Giesselmann, Eur. Phys. J. E 37, 7 (2014).

[19] J. Lagerwall, G. Scalia, M. Haluska, U. Dettlaff-Weglikowska, S. Roth, and F. Giesselmann, Adv. Mater. 19, 359 (2007).

[20] S. H. Aboutalebi, M. M. Gudarzi, Q. B. Zheng, and J. K. Kim, Adv. Funct. Mater. 21, 2978 (2011).

[21] J. E. Kim, T. H. Han, S. H. Lee, J. Y. Kim, C. W. Ahn, J. M. Yun, and A. O. Kim, Angew. Chem. Int. Ed. 50, 3043 (2011).

[22] S. Al-Zangana, M. Iliut, M. Turner, A. Vijayaraghavan, and I. Dierking, Adv. Opt. Mater. 4, 1541 (2016).

[23] J. Mirzaei, M. Urbanski, K. Yu, H. S. Kitzerow, and T. Hegmann, J. Mater. Chem. 21, 12710 (2011).
[24] H. K. Bisoyi and S. Kumar, Chem. Soc. Rev. 40, 306 (2011).

[25] S. Fraden, G. Maret, and D. L. D. Caspar, Phys. Rev. E 48, 2816 (1993).

[26] I. Dierking, W. Blenkhorn, E. Credland, W. Drake, R. Kociuruba, B. Kayser, and T. Michael, Soft Matter 8, 4355 (2012).

[27] W. Weiler, Z. Phys. Chem. Unterricht. 6, 194 (1893).

[28] G. Quincke, Ann. Phys. Chem. 11, 27 (1896).

[29] R. W. O'Brian and L. R. White, J. Chem. Soc. Faraday Trans. 74, 1607 (1978).

[30] C. S. Mangelsdorf and L. R. White, J. Chem. Soc. Faraday Trans. 88, 3567 (1992).

[31] B. J. Yoon and S. Kim, J. Colloid Interface Sci. 128, 275 (1988).

[32] Y. Solomentsev and J. L. Anderson, J. Fluid Mech. 279, 197 (1994).

[33] D. Long and A. Ajdari, Phys. Rev. Lett. 81, 1529 (1998).

[34] B. J. Yoon, J. Colloid Interface Sci. 142, 575 (1991).

[35] Y. E. Solomentsev, Y. Pawar, and J. L. Anderson, J. Colloid Interface Sci. 158, 1 (1993).

[36] T. B. Jones, IEEE Trans. Ind. Appl. IA-20, 845 (1984).

[37] I. Turcu, J. Phys. A 20, 3301 (1987).

[38] G. Cordoyiannis, V. S. Rao Jampani, S. Kralji, S. Dhara, V. Tzitzios, G. Basina, G. Nounesis, Z. Kutnjak, C. S. Pati Tripathi, P. Losada-Perez, D. Jesenek, C. Glorieux, I. Musevic, A. Zidansek, H. Ameinitsch, and J. Thoen, Soft Matter 9, 3956 (2013).

[39] T. Togo, K. Nakayama, M. Ozaki, and K. Yoshino, Jpn. J. Appl. Phys. 36, L1520 (1997).

[40] K. Nakayama, M. Ozaki, and K. Yoshino, Mol. Cryst. Liq. Cryst. 329, 129 (1999).

[41] I. Dierking, P. Cass, K. Syres, R. Cresswell, and S. Morton, Phys. Rev. E 76, 021707 (2007).

[42] I. Dierking, F. Giesselmann, P. Zugenmaier, W. Kuczynski, S. T. Lagerwall and B. Stebler, Liq. Cryst. 13, 45 (1993).

[43] T. A. Wood, A. Wright, H. F. Gleeson, M. Dickinson, T. Mullin, and A. Murray, Proc. SPIE 4634, 128 (2002).

[44] A. Wright, T. A. Wood, M. Dickinson, H. F. Gleeson, T. Mullin, and A. Murray, Proc. SPIE 4622, 195 (2002).

[45] A. K. Srivastava, M. Kim, S. M. Kim, M. K. Kim, K. Lee, Y. H. Lee, M. H. Lee, and S. H. Lee, Phys. Rev. E 80, 051702 (2009).

[46] S. Hernandez-Navarro, P. Tierno, J. Ignes-Mullol, and F. Sagues, Soft Matter 9, 7999 (2013).

[47] O. D. Lavrentovich, I. Lazo, and O. P. Pishnyak, Nature (London) 467, 947 (2010).

[48] A. V. Ryzhkova, F. V. Podgornov, and W. Haase, Appl. Phys. Lett. 96, 151901 (2010). 